\title{
Comparison of two methods for assessment of Microvolt T-Wave Alternans: discrete vs continuous T-wave analysis
}

\author{
Thaís Winkert ${ }^{1}$, Jurandir Nadal $^{1}$, Paulo Roberto Benchimol-Barbosa ${ }^{1,2}$ \\ ${ }^{1}$ Biomedical Engineering Program, COPPE, Universidade Federal do Rio de Janeiro, RJ, Brasil \\ ${ }^{2}$ Hospital Universit ario Pedro Ernesto, Universidade do Estado do Rio de Janeiro, RJ, Brasil
}

\begin{abstract}
Micro T-wave alternans (MTWA) is a risk marker for life threatening ventricular tachyarrhythmia. Classically, MTWA is assessed by quantification of beat-by-beat Twave amplitude alternation. This method requires accurate determination of $T$-wave peaks (discrete method) to create a sequence of T-wave amplitude series, which undergoes spectral analysis using the fast Fourier transform (FFT) for analysis. The need of precise measurements of T-wave amplitudes turns this method sensitive to noise. To overcome this limitation, a new method was developed, based on Hilbert Transform of the T-wave morphology series (continuous method). To perform this task, a $300 \mathrm{~ms}$ window containing the T-wave of every beat was isolated, and concatenated to form an artificial and continuous signal with T-waves. The Hilbert Transform was then applied to calculate the envelope of this signal. The alternans was detected over the envelope FFT, as a peak on the frequency that corresponded to half of the main signal frequency. Both methods were tested in 50 ECG signals of Physionet T-Wave Alternans Database, 31 synthesized and 19 real world signals. The comparisons of the methods were carried out by linear correlation test, Wilcoxon test, and Bland-Altman charts, in channels 1 and 2. There was no significant differences between both methods in all tests, either synthesized or real world signals. The novel continuous method to quantify micro T-wave alternans based on T-wave morphology is feasible, accurate and reproducible, and have potential clinical application.
\end{abstract}

\section{Introduction}

Microvolt T-Wave Alternans (MTWA) is defined as tenuous beat-by-beat variation on T-waves amplitude. T-wave expresses how ventricles repolarizes and represents transmural action potential phase 3 gradient across ventricle wall [1]. On its turn, MTWA can be regarded as beatby-beat transmural phase 3 gradient variation associated with impaired intra-sarcoplasmatic calcium influx and ef- flux dynamics [2,3]. Detection of abnormal MTWA is considered a robust risk marker for life threatening ventricular tachyarrhythmia [4]. The discrete MTWA method has been introduced in early 90's and it is classically quantified by Fourier Transform of sequential T-waves peaks amplitude series. It requires accurate determination of Twaves fiducial points. Therefore, appropriate assessment of T-wave amplitudes is a requirement for MTWA quantification.

However, T-wave amplitude determination may be challenging due to its morphological aspects allied to interference from external sources. For instance, T-wave morphology undergoes continuous variation as a consequence of respiratory-derived amplitude modulation, power line and muscular interferences.

A method for MTWA analysis that does not require Twave peak determination may be a suitable alternative to overcome limitation related to precise $\mathrm{T}$-wave amplitude detection. This study introduces a continuous T-wave amplitude analysis, based on application of the Hilbert Transform on isolated T-wave series, and performs the comparison of current method (continuous method) with the classical MTWA beat-by-beat detection (discrete method).

\section{Methods}

\subsection{The Physionet signals}

The analyzed signals were downloaded from T-Wave Alternans Database, available at PhysioNet website [5]. It contains multichannel ECG records from several collaborative institutions, sampled at $500 \mathrm{~Hz}$ with 16 bit resolution over a $\pm 32 \mathrm{mV}$ range. The database contains 100 signals, 32 of them were synthesized and the remaining 68 were real world ECG signals, from patients with myocardial infarctions, transient ischemia, ventricular tachyarrhythmias, and other risk factors for sudden cardiac death and healthy controls. Most of the signals available had 12 channels ECG. However, some signals were extracted from MIT-BIH Normal Sinus Rhythm Database, Sudden Cardiac Death Holter Database and Long-Term ST 
Database, in which only 2 or 3 ECG leads were available. To make the analysis uniform for all ECG available in this database, it was arbitrarily defined that only the first and the second channels would be analyzed in this study, for all ECG databases.

\subsection{Preprocessing}

The first approach was to preprocess each ECG signal aiming at reducing interference, correcting baseline drifting and discontinuities for improving the correct identification of alternans. Preprocessing consisted in a three step process:

1. Identification of discontinuities - One signal showed short periods of interruption of ECG signal, evidencing either acquisition saturation or interrupted data acquisition. In this case, samples were interpolated to fill all missing data and to recover the ECG. The algorithm inserted in the non-existing data segment the immediate previous existing value in a recursive procedure. However, this signal was further discharged for excessive noise;

2. Low-Pass Filter - After correcting discontinuities, the signal was low-pass filtered (Butterworth, second order, cutoff frequency on $30 \mathrm{~Hz}$ ) to reduce interference;

3. Correction of baseline drifting - The ECG was analyzed to detect R-waves using the absolute first order derivative method. The points at $220 \mathrm{~ms}, 200 \mathrm{~ms}$ and $80 \mathrm{~ms}$ before the peak of the detected R-wave were defined as baseline reference to the respective heart beat. Those three points were identified throughout the ECG data and a cubic spline was fitted to those points over the whole ECG. In order to correct drifting the resulting cubic spline was subtracted from the ECG.

Those procedures provided a corrected output ECG in witch MTWA analysis was carried out. Additionally, on corrected ECG, ectopic beats were identified based on prematurity and QRS complex waveform comparisons.

\subsection{MTWA using the discrete method}

The discrete method consisted of detecting the T-wave peak of every sinus beat in the whole corrected ECG. The peak of the T-wave was detected in a 300 ms width window, starting at $100 \mathrm{~ms}$ after R-wave peak. The T-wave peak of every beat was stored in a vector. MTWA was assessed in a series of T-waves of 128 consecutive sinus beats, ensuring the absence of ectopic rhythms in between. Signals that did not present at least 128 consecutive T-wave peaks were discharged from analysis. T-wave peak series was then Fourier transformed and the peak on the PSD spectra at 0.5 cycles per beat measured [6]. Alternans was calculated as 'alternans ratio', defined as the measured alternans peak divided by the standard deviation of ten consecutive harmonics immediately neighbor to the peak. 'Al- ternans ratio' above three units are considered abnormal [5].

\subsection{MTWA using the continuous method}

The continuous method is an alternate proposition to analyze MTWA. After preprocessing, the earlier described $300 \mathrm{~ms}$ windows were concatenated to form an artificial signal containing consecutive isolated T-waves morphologies. The T-wave signal was clipped below its mean value to reduce its amplitude. A continuous signal was assembled with 128 consecutive isolated T-waves, to allow comparisons with the discrete method.

The Hilbert Transform was applied to the signal composed by the T-wave morphology series, to identify the envelope of the signal, given by the absolute value the of sum of the actual signal and its Hilbert Transformed version multiplied by i. It was hypothesized that if any Twaves peak amplitude variation particularly in a beat-bybeat fashion have MTWA information, the envelope will also carry this information. Therefore, a MTWA will also be captured by the envelope.

In order to isolate an eventual T-wave peak amplitude alternation, the envelope function was Fourier transformed. The peak at the frequency corresponding to half of the whole $\mathrm{T}$-wave series frequency (inverse of the whole $\mathrm{T}$ wave duration interval) was isolated and quantified, defining alternans peak.

Similarly to the discrete method, alternans was calculated as 'alternans ratio', defined as the alternans peak divided by the standard deviation of ten consecutive harmonics immediately neighbor to the peak. 'Alternans ratio' above three units are considered abnormal.

\subsection{Statistical analysis}

Continuous variables were presented as median and interquartile range. Discrete variables were presented as percentile or absolute values, as needed. The methods were compared by Willcoxon test and Bland-Altman charts. To offer a quantitative assessment of Bland-Altman chart significance, the percentage of point outside of the interval of $95 \%$ CI was compared against zero percentage using a Chi-squared method. The alternans peaks were also compared by linear correlation using the squared Pearson correlation coefficient. Alpha error level was set at 0.05 .

\section{Results}

From the 68 real signals, 13 were too short (did not convey 128 consecutive normal beats, at least) and were excluded from the analyses. Additionally, 11 signals were excluded due to excessive baseline noise by visual inspection (Excessive noise group median $0.092 \mu \mathrm{V}$, [lower quar- 
tile $0.049 \mu \mathrm{V}$; upper quartile $0.264 \mu \mathrm{V}])$. The remaining signals accepted for analysis have median $0.009 \mu \mathrm{V} ; \mathrm{p}<$ 0.05 .

All the remaining signals were adequately preprocessed and further analyzed to exclude ectopic beats. After identification of ectopic beats, additional nine signals were excluded, since no sequence of 128 consecutive sinus beats could be achieved. Thus, 19 real and 31 synthesized signals were used for evaluation.

Figure 1 shows the preprocessing stages of a conveniently chosen signal, in channel 1.

Figure 2 illustrate both methods regarding alternans quantification steps for channel 1 on case TWA06, respectively. In continuous method, alternans frequency was found exactly at half of the corresponding whole T-wave series frequency.

The numeric comparison of both methods was performed by boxplots (Figure 3). Bland-Altman chart for peak alternans comparison of both channel 1 and 2 presented only three cases of 50 outside the $95 \%$ confidence interval (Figure 4 and 5). Regression analyses are presented in Figure 6 and 7. Non significant differences between both methods were found after Bland-Altman chart analyses for channels 1 and 2 (respectively, $\chi^{2}=0.017$; $\left.\mathrm{p}=0,87, \chi^{2}=0.04 ; \mathrm{p}=0.83\right)$. Correlation coefficient for channels 1 and 2 were respectively $0.910(\mathrm{p}>0.001)$ and $0.841(\mathrm{p}>0.001)$.

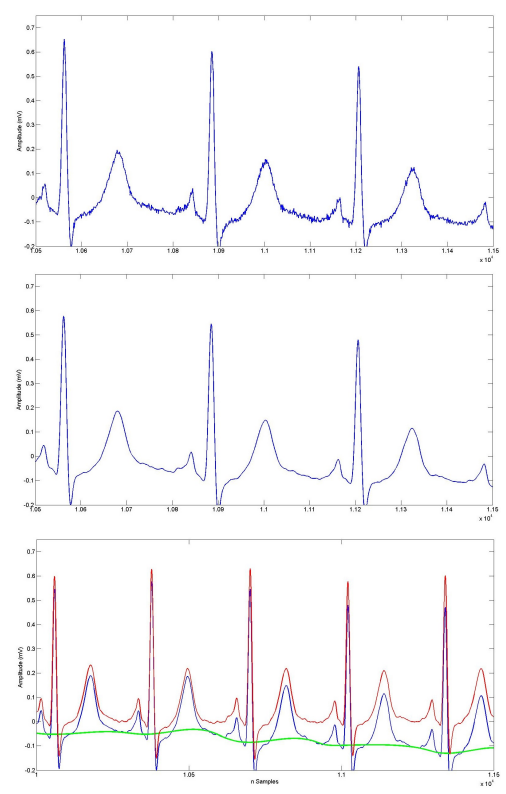

Figure 1. Preprocess results from signal TWA11. Upper panel shows the raw signal of Channel 1 . The middle panel shows the same signal after low pass filtering. The lower panel shows the signal after baseline drifting correction.

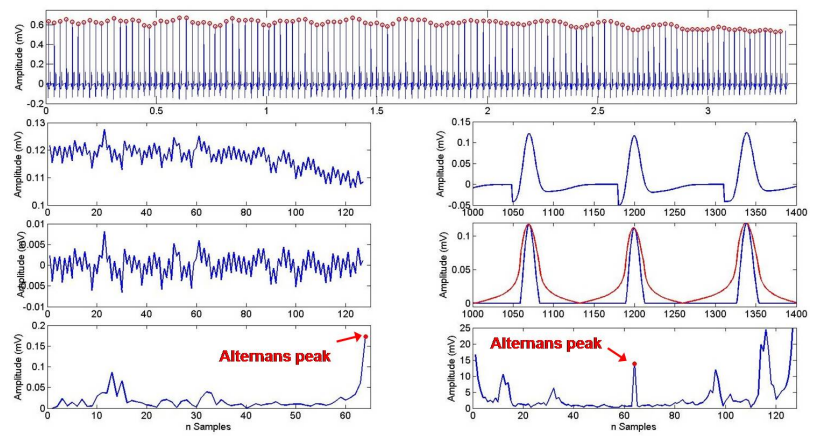

Figure 2. MTWA identification - TWA09 - Channel 1. The upper panel show the signal and the identification of 128 QRS complex. The left panels show the discrete method, respectively: alternans vector, alternans vector without trends and FFT of alternans vector. Right panels demonstrate the continuous method, receptively: T-wave sequence, T-wave sequence, Hilbert transform derived envelope and FFT of the envelope. The arrows indicate the alternans peaks.

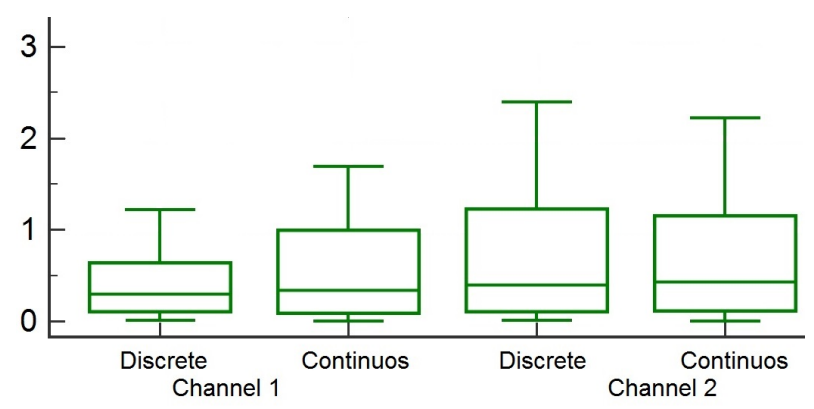

Figure 3. Box-plot for methods numeric comparison. Upper panel shows results for channel 1. Lower panel shows the results for channel 2 .

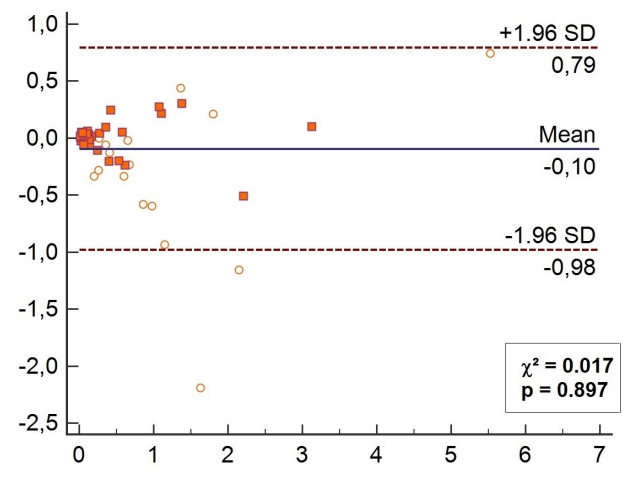

Figure 4. Bland Altman Chart for Channel 1. The square point represents the synthesized data and the open circle represents the real word signals. 


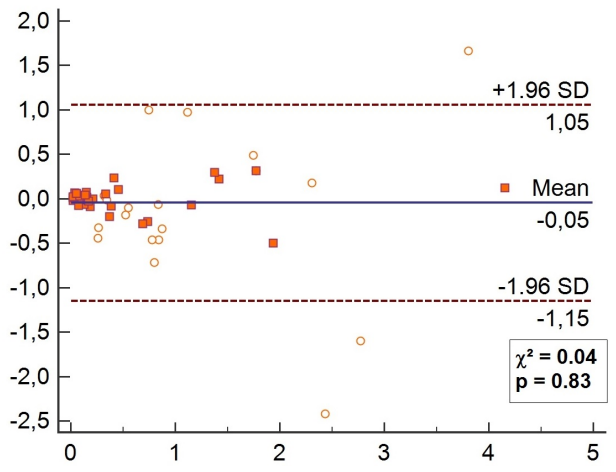

Figure 5. Bland Altman Chart for Channel 2. The square point represents the synthesized data and the open circle represents the real word signals.

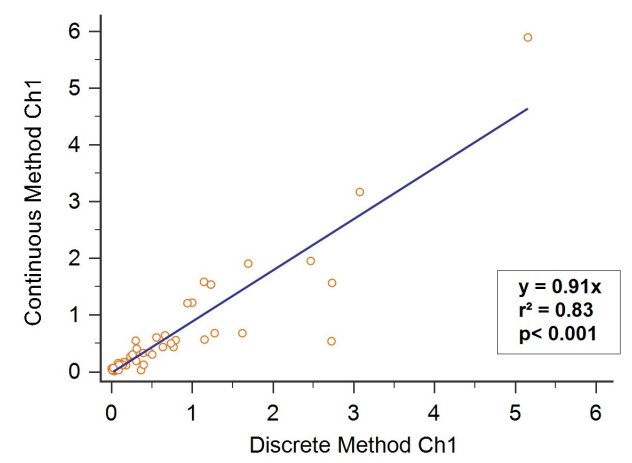

Figure 6. Regression Analyses for Channel 1

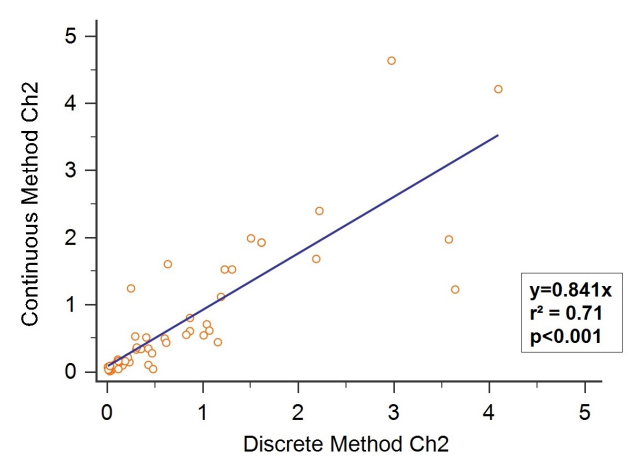

Figure 7. Regression Analyses for Channel 2

\section{Discussion}

Proper risk classification of patients at risk to develop fatal cardiac arrhythmias is needed for prophylaxis and treatment. MTWA is a promising tool for arrhythmia risk stratification. This study describes a method for micro T-wave alternans identification, with no need to precisely isolate Twave peaks. Considering that T-wave peak measure may be affected by external features, such as noise and plateau variations, detection precision may vary beat-by-beat, potentially affecting alternans quantification.

The proposed method uses the envelope of consecutively whole T-waves morphologies for analysis, calculated by Hilbert transform. It allow to analyze a continuous modulator signal, thus avoiding the need of accurate T-wave peaks detection. The present method, applied to a standard database containing T-wave alternans ECG tracings, available at Physionet, shows high numeric correlation and reproducibility with classical method. Therefore, it turns to be a promising tool for clinical application.

Potential limitations of present method are the requirement of T-wave morphology clipping, that may influence the amplitude of the Hilbert transform envelope. In subjects with flat T-waves, the detection of alternans may be compromised, making MTWA indeterminate. This study was based on numeric comparison of normalized alternans values. Further clinical validation based on alternans classification is needed.

As a conclusion, the continuous method to quantify micro T-wave alternans based on whole T-wave morphology analysis is feasible, accurate and reproducible, and have potential clinical application.

\section{References}

[1] Smith, JM, et al. Electrical alternans and cardiac electrical instability. Circulation 1988;77:110-121.

[2] Akar FG, et al. Transmural Electrophysiological Heterogeneities Underlying Arrhythmogenesis in Heart Failure. Circ Res. 2003;93:638-645.

[3] Walker ML,et al. Hysteresis Effect Implicates Calcium Cycling as a Mechanism of Repolarization Alternans. Circulation. 2003;108:2704-2709.

[4] Benchimol-Barbosa PR,et al. Alternâcia elétrica da onda T: Bases eletrofisiológicas e aplicações clínicas baseadas em evidências. [In Portuguese] Revista da SOCERJ 2004; 17:227242.

[5] Detecting and Quantifying T-Wave Alternans. Physionet. www.physionet.org/challenge/2008/ assessed on April, 15th, 2016.

[6] Rosenbaum, DS et al. Eletrical alternans and vulnerability to ventricular arrhythmias. The New England Journal of Medicine 1994;330:235241.

Address for correspondence:

Jurandir Nadal

Programa de Engenharia Biomédica - COPPE/UFRJ

PO Box 68510

Rio de Janeiro 21941-97

jn@peb.ufrj.br 